\title{
ERRATUM
}

\section{Quantitative analysis of ipsilateral and contralateral supracerebellar infratentorial and occipital transtentorial approaches to the cisternal pulvinar: laboratory anatomical investigation}

TO THE READERSHIP: An error appeared in the article by Sun et al. (Sun Q, Zhao X, Gandhi S, et al. Quantitative analysis of ipsilateral and contralateral supracerebellar infratentorial and occipital transtentorial approaches to the cisternal pulvinar: laboratory anatomical investigation. J Neurosurg. Published online August 2, 2019. doi:10.3171/2019.4.JNS19351).

The key in Fig. 1A was incorrect. The revised image is shown here with the corrected key. 2020.

The article has been corrected online as of August 14,

Mark C. Preul, MD

Barrow Neurological Institute, St. Joseph's Hospital and Medical Center,

Phoenix, AZ

CORRESPONDING ARTICLE See pp 1172-1181.

INCLUDE WHEN CITING

Published online August 14, 2020; DOI: 10.3171/2020.8.JNS19351a.

CAANS 2020, except where prohibited by US copyright law
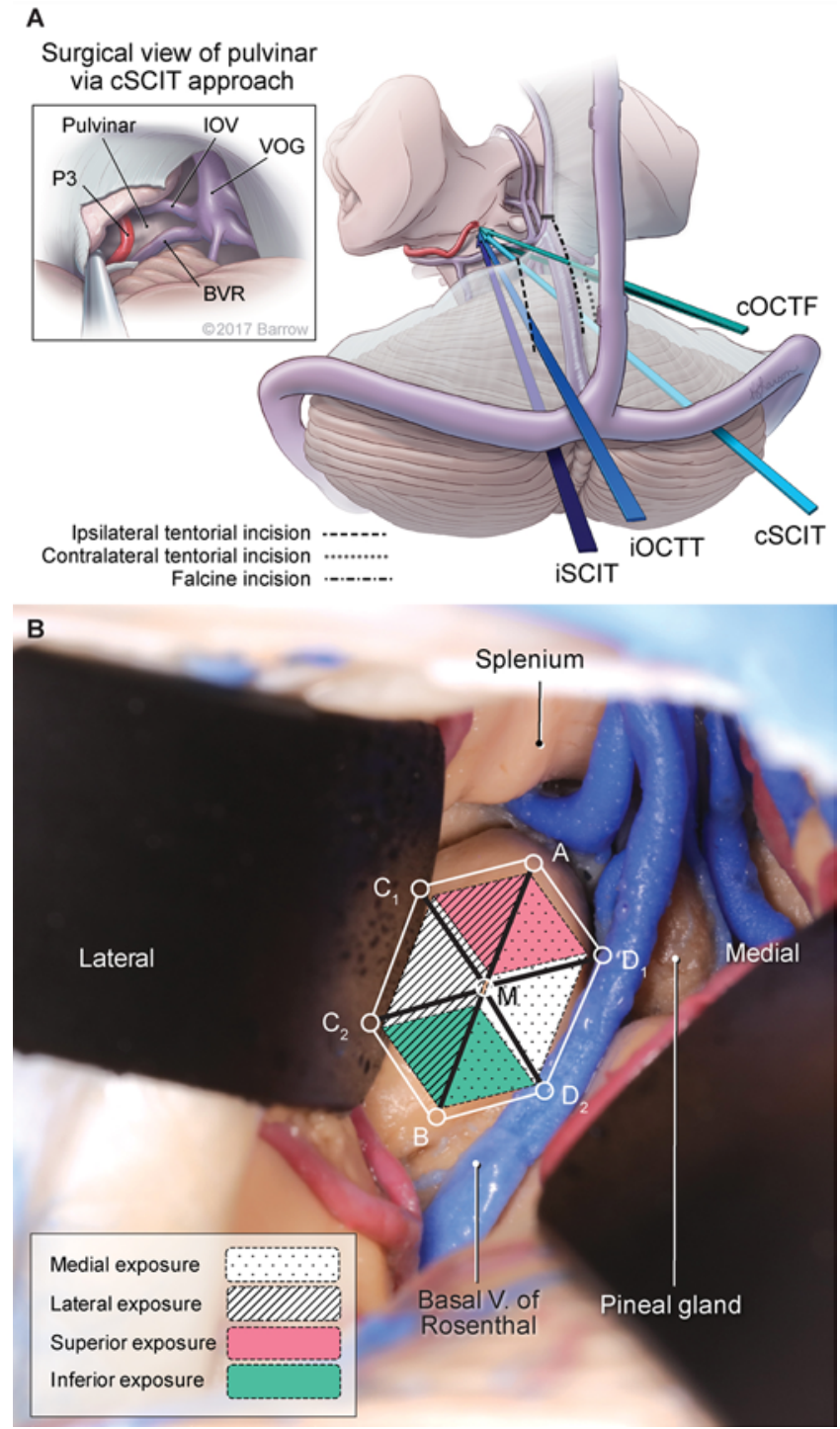

FIG. 1. A: The trajectories for the iSCIT, cSCIT, IOCTT, and COCTF approaches to the pulvinar. The head is placed in the prone position for occipital approaches. In the iOCTT approach, a 2-cm tentorial incision is made lateral to the straight sinus on the ipsilateral side. In the cOCTF approach, in addition to the tentorial incision made ipsilateral to the craniotomy, a $2.5-\mathrm{cm}$ falcine incision is made superior to the straight sinus, with transection of the inferior sagittal sinus. The straight sinus can be mobilized either superiorly or inferiorly to offer inferior or superior trajectories, respectively. A sitting position is used to secure the head for supracerebellar approaches. B: Illustration of the measurement of the area of exposure from point M, the most posteriorly prominent point on the cisternal pulvinar. $A 6$-sided anatomical polygonal region $\left(A C_{1} C_{2} B D_{2} D_{1}\right)$ is configured over the cisternal pulvinar. Point $A$ is the superior reachable point and point $B$ is the inferior reachable point on an imaginary line along the long axis of the pulvinar through point $M$. Points $C_{1}$ and $C_{2}$ are the superior and inferior reachable points on the lateral edge, respectively, and points $D_{1}$ and $D_{2}$ are the superior and inferior reachable points on the medial edge, respectively. The sum of the areas of the 6 triangles $\left(A C_{1} M, C_{1} C_{2} M, C_{2} B M, B D_{2} M, D_{2} D_{1} M\right.$, and $\left.D_{1} A M\right)$ represents the total area of pulvinar exposure. The medial area of exposure is the sum of the areas of triangles $A D_{1} M, D_{1} D_{2} M$, and $D_{2} B M$ (dotted area). The lateral area of exposure is the sum of the areas of triangles $A C_{1} M, C_{1} C_{2} M$, and $C_{2} B M$ (hatched area). The superior area of exposure is the sum of the areas of triangles $A C_{1} M$ and $A D_{1} M(r e d)$. The inferior area of exposure is the sum of the areas of triangles $B C_{2} M$ and $B D_{2} M(g r e e n)$. $P 3=$ third segment of the $P C A ; V O G=$ vein of Galen. Used with permission from Barrow Neurological Institute, Phoenix, Arizona. Figure is available in color online only. 